\title{
Recent Research Work on Advanced Materials Processing
}

\author{
Deliang Zhang ${ }^{a^{*}}$ (D), Gaowu Qin ${ }^{a}$ \\ ${ }^{a}$ Key Laboratory for Anisotropy and Texture of Materials (Ministry of Education), School of Materials \\ Science and Engineering, Northeastern University, Shenyang 110819, China
}

Received: November 12, 2019; Accepted: November 13, 2019

This ICAMP-9 special volume of Materials Research collects 23 high quality research papers which have been presented at the $9^{\text {th }}$ International Conference on Advanced Materials Processing (ICAMP-9) held on September 25-28, 2018 at Northeastern University in Shenyang, China. Delegates who presented papers at the conference were invited to submit manuscripts for consideration for publishing in the ICAMP-9 special issue of Materials Research. The manuscripts were first submitted to the conference organizing committee and screened to select those which have sufficiently high quality to be recommended to Materials Research. Then each of the recommended and submitted manuscripts was reviewed by two expert reviewers according to the academic standard of the journal. Most of the submitted manuscripts were substantially revised according to the reviewers' comments, and eventually 23 manuscript have been finalized and accepted for publication in the ICAMP-9 special issue.

Although the number of the papers in the special issue is not large, they nicely reflect the polarization of the research interests and content in the area of advanced materials processing. They encompass materials for structural and functional applications, and research focus in microstructural evolution and processing-microstructure-property relationships. In terms of the materials types, the papers cover metallic materials, ceramic materials and other types of inorganic materials. Since the conference is biased towards metallic and non-metallic inorganic materials, there is no papers on polymers in this special volume. In the modern era of advanced materials research, researchers need to have a vision in the commonality of a variety of materials exhibiting different microstructures and properties, and being studied for various applications. That is: in any advanced materials and related materials processing technologies, we will see the importance of underlying processing-microstructure-properties relationships which are governed by physical laws such as thermodynamics and micromechanics.

We thank the authors for the contributions of the high quality work and the reviewers for their tireless effort in reviewing the manuscripts and making recommendations and comments. It is expected that the 23 high quality research papers in the ICAMP-9 special issue of Materials Research will make important contributions to the research area of advanced materials processing. 